\title{
Venous function after pharmaco-mechanical thrombolysis for extensive ileofemoral deep vein thrombosis: Six-year outcome
}

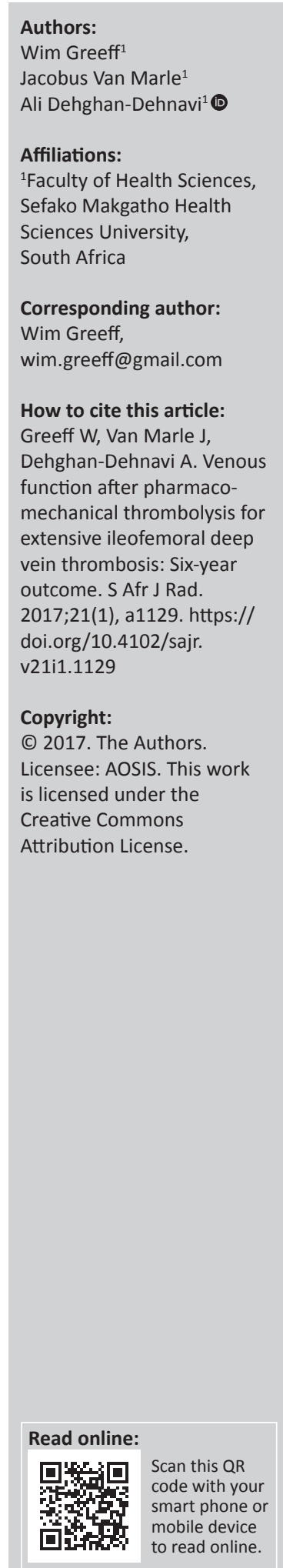

Background: Chronic venous insufficiency is an important complication following ileofemoral deep venous thrombosis. Early thrombus removal may preserve venous function and prevent this complication.

Objective: To evaluate the long-term outcome following pharmaco-mechanical thrombolysis (PMT) for proximal deep venous thrombosis in a private, specialist vascular unit.

Methods: All patients who had undergone PMT for ileofemoral deep venous thrombosis (IFDVT) between August 2009 and January 2016 were invited to return for clinical assessment and venous ultrasound study. Clinical findings were recorded according to the Villalta score and clinical, etiological, anatomical and pathological (CEAP) classification. Quality of life (QOL) was assessed according to the VEnous INsufficiency Epidemiologic and Economic Study (VEINES) QOL/Sym Questionnaire.

Results: A total of 32 patients (35 legs) were evaluated. There were 25 females and 7 males with a mean age of 33.5 years. The mean follow-up period was 31 months.

Results: Twenty-four patients (75\%) were C0, C1 (1), C2 (2), C3 (2) and C4 (3). With the Villalta score, 28 patients had mild or no evidence of venous disease (scores 0,1 or 2). Four patients had moderate symptoms (scores 3-6). With the VEINES questionnaire, the results were good with the scores for QOL ranging between 0.30 and 0.60 and the Sym range being $0.32-0.61$. The venous ultrasound only found four patients to have mild fibrosis with reflux.

Conclusion: Patients who had undergone PMT for extensive IFDVT showed no significant clinical signs of chronic venous insufficiency, had excellent function on venous ultrasound and reported excellent QOL.

Note: A selection of conference abstracts: RSSA/SASPI Paediatric Imaging Congress, 03-06 November 2016, Spier Estate, Stellenbosch, South Africa. Faculty collaborators: Professor Kassa Darge (Body Imaging, University of Pennsylvania, Philadelphia, USA), Professor Edward Lee (TSoracic Imaging, Harvard University, USA), Professor Beverley Newman (Cardiac Imaging, Stanford University, California, (Th), Prof (Thoracic Imaging, University of Bristol, UK) supported by South African Paediatric Radiologists, co-ordinated by Dr. Jaishree Naidoo, President of the African Society of Paediatric Imaging and Head of Division of Paediatric Radiology, Charlotte Maxeke Johannesburg Academic Hospital. 